\title{
Anxiety as a risk factor in coronary artery disease among young women
}

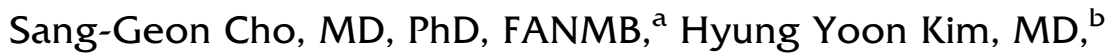 \\ and Hee-Seung Bom, MD, PhD, FANMB ${ }^{\mathrm{C}}$ \\ a Department of Nuclear Medicine, Chonnam National University Hospital, Gwangju, Republic of \\ Korea \\ b Department of Cardiology, Chonnam National University Hospital, Gwangju, Republic of Korea \\ c Department of Nuclear Medicine, Chonnam National University Hwasun Hospital, Hwasun- \\ gun, Jeonnam, Republic of Korea
}

Received Mar 5, 2020; accepted Mar 5, 2020

doi: $10.1007 / \mathrm{s} 12350-020-02101-8$

\begin{tabular}{|c|c|c|c|c|}
\hline \multicolumn{3}{|c|}{ Abbreviations } & \multirow{2}{*}{$\begin{array}{l}\text { GAD } \\
\text { FMM }\end{array}$} & \multirow{2}{*}{$\begin{array}{l}\text { Generalized anxiety disorder } \\
\text { Fractional myocardial mass }\end{array}$} \\
\hline $\begin{array}{l}\text { CAD } \\
\text { SPECT }\end{array}$ & $\begin{array}{l}\text { Coronary artery disease } \\
\text { Single-photon emission } \\
\text { tomography }\end{array}$ & computed & & \\
\hline
\end{tabular}

See related article, pp. 2581-2592

\section{INTRODUCTION}

Coronary artery disease (CAD) in women has several distinctive features from that in men. In addition to the traditional risk factors (e.g., diabetes, smoking), there are some 'non-traditional' risk factors for CAD in women including preterm delivery, gestational diabetes, gestational hypertension, autoimmune disease, and notably, psychiatric disorders. ${ }^{1}$ Psychiatric disorders are not rare among patients with CAD and considered to be linked with CAD in pathophysiological aspects. ${ }^{2,3}$ The most highlighted psychiatric disorder regarding CAD is depression, which has already been extensively investigated and integrated into the clinical guidelines. ${ }^{4,5}$ Anxiety disorders have gained less attention than depression, but are getting more clinical relevance. ${ }^{6}$

In this issue of the Journal of Nuclear Cardiology, Bekendam et al. ${ }^{7}$ demonstrated an intriguing association

\footnotetext{
Reprint requests: Hee-Seung Bom, MD, PhD, FANMB, Department of Nuclear Medicine, Chonnam National University Hwasun Hospital, 322, Seoyang-ro Hwasun-eup, Hwasun-gun, Jeonnam 58128, Republic of Korea; hsbom@jnu.ac.kr

J Nucl Cardiol 2021;28:2593-6.

1071-3581/\$34.00

Copyright (C) 2020 American Society of Nuclear Cardiology.
}

between anxiety and the severity of myocardial ischemia, which may explain the potential link between anxiety and poorer prognosis of CAD among women. They analyzed the association between anxiety and myocardial ischemia in relation to two demographic factors, gender and age. Trait anxiety was associated with severer myocardial ischemia on myocardial perfusion single-photon emission computed tomography (SPECT), which was significant only for younger women ( $\leq 65$ years old). Younger women with high trait anxiety (General Anxiety Disorder [GAD]-7 score $>10$ ) showed significantly severer myocardial ischemia as compared to those with low trait anxiety (GAD-7 score $\leq 10$ ).

Anxiety exhibits chronic withdrawal of parasympathetic activity. ${ }^{8}$ As parasympathetic innervation mainly determines the chronotropic aspect of the heart, there is a significant decrease in high-frequency heart rate variability in patients with anxiety disorders. The high-frequency domain of heart rate variability represents the parasympathetic controls on the cardiac rhythm, and its impairment is associated with major symptoms of anxiety disorders-hypervigilance and worry. In addition, the impaired parasympathetic activity leads to impaired 


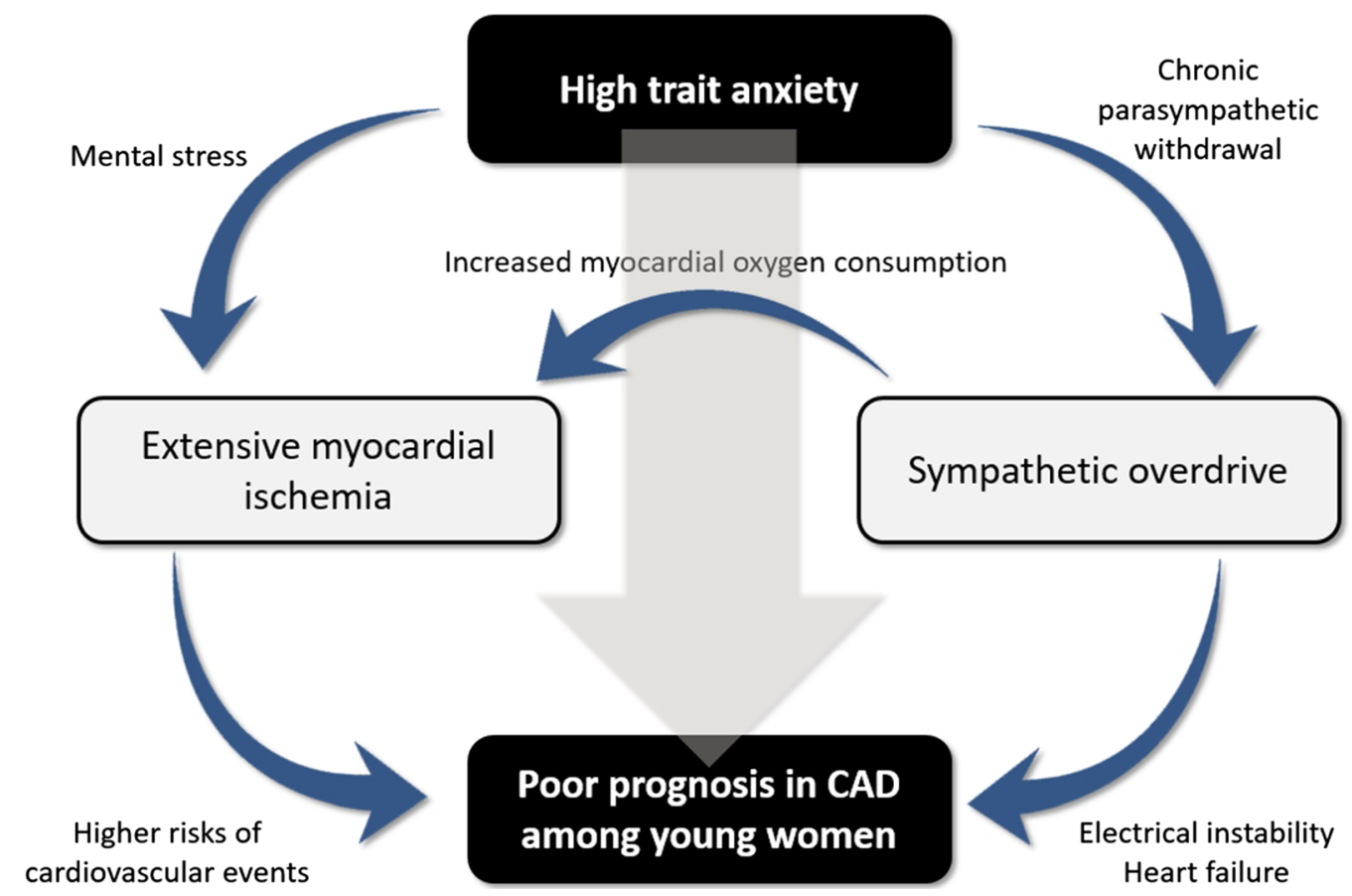

Figure 1. Mechanisms explaining the link between anxiety and poorer prognosis in CAD among women. Anxiety shows chronic withdrawal of parasympathetic activity, which induces sympathetic overdrive. This leads to exaggerated myocardial ischemia due to increased myocardial oxygen consumption by more prominent hemodynamic reactivity. At the same time, electrical instability due to sympathetic overdrive raises vulnerability to the incidence of heart failure. Anxiety itself may cause myocardial ischemia via mental stress. $C A D$ coronary artery disease.

sympatho-excitatory inhibition. This might have contributed to the prominent chronotropic response to stress testing in patients with high trait anxiety in the present study. More marked increase in heart rate inevitably results in higher myocardial oxygen demand, which could exaggerate the extent and severity of myocardial ischemia despite similar severity of coronary artery disease. ${ }^{9}$

It is noteworthy that trait anxiety, not state anxiety, was associated with more prominent hemodynamic reactivity to stress testing and myocardial ischemia. State anxiety is defined as transitory anxious behaviors, cognitions, and/or affects that result from an interaction of person characteristics and situational influences. ${ }^{10}$ State anxiety in the present study was evaluated with a novel artificial intelligence technique, the FaceReader. ${ }^{11}$ It read the facial expressions of anxiety to detect the timely evidences of state anxiety before stress, during stress, and at the recovery phase. However, neither increased hemodynamic reactivity nor demographic difference (gender or age) was associated with the presence of state anxiety. State anxiety did not affect the severity of myocardial ischemia, either. Instead, high trait anxiety was associated with increased hemodynamic reactivity and more extensive myocardial ischemia. As trait anxiety is defined as a chronic, stable tendency to respond fearfully to a wide variety of unspecific stressors, ${ }^{12}$ the anxiety-ischemia association should not be regarded as a transient, momentary phenomenon due to the nervous mood resulting from SPECT imaging and stress testing, so-called white-coat phenomenon.

Then, why was the anxiety-ischemia association found only for younger women? Aging is related to a decreased sympathetic reactivity to stress testing. Although the sympathetic activity is generally elevated (e.g., higher resting systolic blood pressure and heart rate), the sensitivity of the adrenergic receptors to sympathetic stimulation is declined in elderly patients. Thus, the physiologic increases in heart rate, cardiac output and vasodilation in response to adrenergic stimulation are diminished. ${ }^{13}$ Given the diminished sympathetic reactivity in the elderly people, it is not surprising that anxiety was associated with severe myocardial ischemia only for younger patients. Parallel decrease in anxiety is also noted in the elderly people.

However, the anxiety-ischemia association was not found for younger men, but only for younger women. Such female predisposition to higher hemodynamic 
reactivity to stress testing and inducible myocardial ischemia have been consistently shown in previous studies. ${ }^{14,15}$ A possible explanation can be provided by the biological differences in hormonal homeostasis. The ovarian steroids are thought to control sympathetic drive on the heart and their monthly fluctuations may characterize the sex differences in myocardial ischemia as well as anxiety. ${ }^{16,17}$ Another explanation can be provided by the unique phenotypic features of CAD in women. Women have significantly smaller coronary luminal diameter and the mass of myocardium fed by a specific coronary branch (fractional myocardial mass [FMM]). ${ }^{18,19}$ Despite smaller FMM, the blood flow per unit mass of myocardium is significantly higher for women both at rest and during stress. ${ }^{20}$ Thus, the shear stress against the coronary wall is higher for women. These in combination contribute to the unique CAD characteristics in women: more marked microvascular dysfunction, more common diffuse non-obstructive CAD, plaque erosion, thrombus formation, distal emboli, etc. ${ }^{1}$ Although it is not clear how these features are associated with anxiety, hemodynamic reactivity to stress testing, and the severity of inducible ischemia, the study by Bekendam et al. adds potential explanations to the poorer prognosis of younger women with CAD.

Now, a schematic presentation of the pathophysiology can be built that sheds light on the link between anxiety and poorer prognosis of CAD among women (Figure 1). Anxiety incurs sympathetic overdrive; it directly stimulates cardiac sympathetic tone which can also lead to electrical instability and heart failure; it can also induce extensive myocardial ischemia, which has long been known as a marker of poor cardiovascular outcomes. Anxiety per se may induce myocardial ischemia via mental stress, to which women are particularly vulnerable. ${ }^{21}$ In such perspectives, it is desirable to screen mental health in the initial evaluation of CAD in women, and detect underlying functional impairment even if obstructive CAD is absent. Functional imaging studies (e.g, myocardial perfusion SPECT), will play important roles in the management of $\mathrm{CAD}$ in women.

\section{Acknowledgements}

This work was supported by Basic Science Research Program through the National Research Foundation of Korea (NRF) funded by the Ministry of Education (NRF2016R1D1A3B01006631).

\section{Disclosure}

Sang-Geon Cho and Hyung Yoon Kim report no financial support or conflict of interest. Hee-Seung Bom is supported by a research grant (NRF-2016R1D1A3B01006631) of the Basic Science Research Program through the National Research
Foundation (NRF) funded by the Ministry of Education, Republic of Korea.

\section{References}

1. Garcia M, Mulvagh SL, Merz CNB, Buring JE, Manson JE. Cardiovascular disease in women: clinical perspectives. Circ Res. 2016;118:1273-93.

2. De Hert M, Detraux J, Vancampfort D. The intriguing relationship between coronary heart disease and mental disorders. Dialogues Clin Neurosci. 2018;20:31-40.

3. Schaich A, Westermair AL, Munz M, Nitsche S, Willenborg B, Willenborg C, et al. Mental health and psychosocial functioning over the lifespan of German patients undergoing cardiac catheterization for coronary artery disease. Front Psychiatry. 2018;9:338

4. Lichtman JH, Bigger JT Jr, Blumenthal JA, Frasure-Smith N, Kaufmann PG, Lesperance F, et al. Depression and coronary heart disease: recommendations for screening, referral, and treatment: a science advisory from the American Heart Association Prevention Committee of the Council on Cardiovascular Nursing, Council on Clinical Cardiology, Council on Epidemiology and Prevention, and Interdisciplinary Council on Quality of Care and Outcomes Research: endorsed by the American Psychiatric Association. Circulation. 2008;118:1768-75.

5. Piepoli MF, Hoes AW, Agewall S, Albus C, Brotons C, Catapano $\mathrm{AL}$, et al. European Guidelines on cardiovascular disease prevention in clinical practice: The Sixth Joint Task Force of the European Society of Cardiology and Other Societies on Cardiovascular Disease Prevention in Clinical Practice (constituted by representatives of 10 societies and by invited experts): Developed with the special contribution of the European Association for Cardiovascular Prevention \& Rehabilitation (EACPR). Eur J Prev Cardiol. 2016;23:NP1-96.

6. Celano CM, Millstein RA, Bedoya CA, Healy BC, Roest AM, Huffman JC. Association between anxiety and mortality in patients with coronary artery disease: A meta-analysis. Am Heart J. 2015;170:1105-15.

7. Bakendam MT, Mommersteeg PMC, Kop WJ, Widdershoven JW, Vermeltfoort IAC. Anxiety and hemodynamic reactivity during cardiac stress testing: The role of gender and age in myocardial ischemia. J Nucl Cardiol. 2020. https://doi.org/10.1007/s12350020-02079-3.

8. Chalmers JA, Quintana DS, Abbott MJ, Kemp AH. Anxiety disorders are associated with reduced heart rate variability: A metaanalysis. Front Psychiatry. 2014;5:80.

9. Hoffman JI, Buckberg GD. The myocardial oxygen supply: Demand index revisited. J Am Heart Assoc. 2014;3:e000285.

10. Edmondson D, Shaffer JA, Chaplin WF, Burg MM, Stone AA, Schwartz JE. Trait anxiety and trait anger measured by ecological momentary assessment and their correspondence with traditional trait questionnaires. J Res Pers. 2013. https://doi.org/10.1016/j.jrp. 2013.08.005.

11. Lewinski P. Automated facial coding software outperforms people in recognizing neutral faces as neutral from standardized datasets. Front Psychol. 2015;6:1386.

12. Sehlmeyer C, Dannlowski U, Schoning S, Kugel H, Pyka M, Pfleiderer B, et al. Neural correlates of trait anxiety in fear extinction. Psychol Med. 2011;41:789-98.

13. Hotta H, Uchida S. Aging of the autonomic nervous system and possible improvements in autonomic activity using somatic afferent stimulation. Geriatr Gerontol Int. 2010;10:S127-36. 
14. Gebhard C, Messerli M, Lohmann C, Treyer V, Bengs S, Benz DC, et al. Sex and age differences in the association of heart rate responses to adenosine and myocardial ischemia in patients undergoing myocardial perfusion imaging. $\mathrm{J}$ Nucl Cardiol. 2020;27:159-70.

15. Vaccarino V, Wilmot K, Al Mheid I, Ramadan R, Pimple P, Shah AJ, et al. Sex differences in mental stress-induced myocardial ischemia in patients with coronary heart disease. J Am Heart Assoc. 2016;5:e003630.

16. Souza HC, Tezini GC. Autonomic cardiovascular damage during post-menopause: The role of physical training. Aging Dis. 2013;4:320-8.

17. Hantsoo L, Epperson CN. Anxiety disorders among women: a female lifespan approach. Focus (Am Psychiatr Publ). 2017; 15:162-72.

18. Kim HS, Tonino PA, De Bruyne B, Yong AS, Tremmel JA, Pijls $\mathrm{NH}$, et al. The impact of sex differences on fractional flow reserveguided percutaneous coronary intervention: A FAME (Fractional
Flow Reserve Versus Angiography for Multivessel Evaluation) substudy. JACC Cardiovasc Interv. 2012;5:1037-42.

19. Kang SJ, Ahn JM, Han S, Lee JY, Kim WJ, Park DW, et al. Sex differences in the visual-functional mismatch between coronary angiography or intravascular ultrasound versus fractional flow reserve. JACC Cardiovasc Interv. 2013;6:562-8.

20. Patel MB, Bui LP, Kirkeeide RL, Gould KL. Imaging microvascular dysfunction and mechanisms for female-male differences in CAD. JACC Cardiovasc Imaging. 2016;9:465-82.

21. Vaccarino V, Sullivan S, Hammadah M, Wilmot K, Al Mheid I, Ramadan R, et al. Mental stress-induced-myocardial ischemia in young patients with recent myocardial infarction: sex differences and mechanisms. Circulation. 2018;137:794-805.

Publisher's Note Springer Nature remains neutral with regard to jurisdictional claims in published maps and institutional affiliations. 